\title{
Initial experience with the subcutaneous implantable cardioverter-defibrillator with the real costs of hospitalization analysis in a single Polish center
}

\author{
Marcin Grabowski ${ }^{1}$, Monika Gawałko ${ }^{1}$, Marcin Michalak ${ }^{1}$, Andrzej Cacko ${ }^{1}$, \\ Michał Kowara ${ }^{1,2}$, Agnieszka Kołodzińska ${ }^{1}$, Łukasz Januszkiewicz ${ }^{1}$, \\ Paweł Balsam ${ }^{1}$, Laura Vitali Serdoz ${ }^{3}$, Joachim Winter ${ }^{3}$, Grzegorz Opolski ${ }^{1}$ \\ ${ }^{1} 1^{\text {st }}$ Department of Cardiology, Medical University of Warsaw, Poland \\ ${ }^{2}$ Chair and Department of Experimental and Clinical Pharmacology, Medical University of Warsaw, Poland \\ ${ }^{3}$ Division of Cardiac Surgery, University of Düsseldorf, Germany
}

\begin{abstract}
Background: The recent introduction of an entirely subcutaneous implantable cardioverter-defibrillator (S-ICD) represents an important progress in the defibrillation technology towards a less invasive approach. This is a single-center observational study of S-ICD implantations in Poland.

Methods: The S-ICD was implanted in 11 patients with standard indications for an ICD. Patients in whom the device was implanted were evaluated for adverse events and device function at hospital discharge. All hospitalization costs were calculated and summed up for all patients. Costs were divided into following categories: medical materials, pharmaceuticals, operating theatre staff, cardiology department staff, laboratory tests, non-laboratory tests and additional non-medical costs.

Results: The mean age of patients was $51.6 \pm 16.4$ years, 9 were men and 2 were women. Four patients had atrial fibrillation as the basal rhythm, 1 patient had atrial flutter and 6 patients had sinus rhythm. All patients had at least one condition that precluded the use of a traditional ICD system or the S-ICD was preferred due to other conditions, i.e. a history complicated transvenous ICD therapy (18\%), anticipated higher risk of infection (27\%), lack or difficult vascular access (18\%), young age and anticipated high cumulated risk of lifetime device therapy (36\%). The mean duration of the implantation procedure was $2 \mathrm{~h}$. One patient developed a postoperative pocket hematoma. Mean total time of hospitalization was 28 (6-92) days. Average cost of hospitalization per patient was 21,014.29 EUR (minimal =19,332.71 EUR and maximal = 24,824.14 EUR).

Conclusions: S-ICD implantation appears to provide a viable alternative to transvenous ICD, especially for patients without pacing requirements. (Cardiol J 2019; 26, 4: 360-367)
\end{abstract}

Key words: leadless, primary prevention, procedure cost, secondary prevention, subcutaneous implantable cardioverter-defibrillator, ventricular arrhythmias

\section{Introduction}

Sudden cardiac death (SCD) continues to be a major public health challenge, representing close to one-fifth of all mortality in industrialized countries and claiming about half of its victims not pre- viously diagnosed with heart disease [1]. In recent decades implantable cardioverter-defibrillators (ICDs) have become the treatment of choice in patients with a high risk of fatal ventricular arrhythmia [2]. A weak component of traditionally used ICD systems is the defibrillation lead, which is

Address for correspondence: Marcin Grabowski, MD, PhD, FESC, First Chair and Department of Cardiology,

Medical University of Warsaw, ul. Banacha 1a, 02-097 Warszawa, Poland, tel: +48 22599 19 58, fax: +48 2259919 57,

e-mail: marcin.grabowski@wum.edu.pl

Received: 8.12.2017

Accepted: 12.02.2018 
placed through the venous circulatory system into the right ventricle. It was shown that the longevity of defibrillation leads have become substantially reduced, and risk of infection is significantly higher compared to pacing leads [3]. The subcutaneous implantable cardioverter-defibrillator (S-ICD) was developed in response to endovascular lead complications and represents important progress in defibrillation technology. After obtaining Conformité Européene (CE) marking in 2009 and United States (US) Food and Drug Administration (FDA) approval in 2012, there is growing clinical evidence regarding its safety and efficacy in prevention of SCD [4-11]. This novel technology seems promising and particularly beneficial in patients with conditions associated with higher risk of infections (dialysis, diabetes mellitus) or in those who had experienced previous complications related to transvenous (TV) leads. The clinical benefits of S-ICD therapy have been also documented in young patients facing a lifetime of device therapy $[12,13]$.

The availability of S-ICD therapy is limited in Poland due to a lack of reimbursement and the cost of the device itself is around 7-times higher than conventional transvenous single chamber ICD. Further advancements of this system and cost reduction will likely lead to the expansion of indications and widespread use of this technology. An analysis of the indications was performed, procedural data and the full costs of hospitalization of patients implanted with the S-ICD in single Polish center.

\section{Methods}

\section{Procedure}

The first four implantations were performed with the aid of three different international proctors who had sufficient experience in the procedure. Subsequent S-ICD implant surgeries were performed by two operators from Department of Cardiology of Central Teaching Hospital, Warsaw, Poland. All procedures were performed under general anesthesia. In nine cases, the pocket for the generator was created between the serratus anterior and the latissimus dorsi muscles. In the other 2 cases (case 2 and 3) subcutaneous device placement were used based on proctor advice. The lead was positioned in the subcutaneous tissue of the chest, parallel to and $1-2 \mathrm{~cm}$ to the left sternal midline followed by a perpendicular segment, at the level of the $6^{\text {th }}$ rib, until it reached the pocket of the device. The lead had a $8-\mathrm{cm}$ shock coil, flanked by two sensing electrodes - the distal one positioned close to the manubriosternal joint and the proximal one adjacent to the xiphoid process based on preprocedural X-ray imaging. Immediately after implantation, the most suitable sensing vector (primary, secondary or alternate) was chosen automatically by the S-ICD. The detection was set for two zones (a conditional shock zone and shock zone depending on the patient's indication and condition). Duration of procedure as total implantation time (time from patient in to patient out) and in-hospital adverse events related to the procedure were evaluated.

\section{Patients}

None of the patients had absolute indication for permanent pacing. Prior to the procedure patients were screened with a manual screening tool to assess the applicability of this technology. All the patients were informed of the characteristics of the new system, indications and potential complications. Informed consents were obtained.

\section{Costs of hospitalization analysis}

All costs of hospitalization were calculated and summed up for all patients. Costs were divided into following categories: S-ICD hardware (a device plus a defibrillation lead), medical materials excluding S-ICD (disposable materials related to the procedure, lead introducer), pharmaceuticals (oral drugs, antibiotics, disinfectants, analgesics), operating theatre staff (electrophysiologists, scrub nurse, personal costs of analgesia), cardiology department staff (cardiologists, nurses), additional laboratory tests, additional non-laboratory tests (echocardiography, X-ray), additional non-medical costs (materials and energy, linen, maintenance materials, office supplies, informatics and information technology, laboratory reagents, medical gases, electricity, heat, water, permanent foreign services, postage and telephone charges - non-medical indirect costs, management). Costs are in euro currency, using then current exchange rate (2017-10-06): 1 euro $(E U R)=4.24$ Polish zloty (PLN).

\section{Results}

The S-ICD implantation was attempted in 11 patients with $100 \%$ success rate. All the patients had standard indications for ICD implantation that can be divided into two broad categories: secondary (45.5\% of patients) and primary prophylaxis. For secondary prophylaxis, ICD placement was indicated as initial therapy in survivors of sudden cardiac arrest due to ventricular fibrillation (VF) 
or hemodynamically unstable ventricular tachycardia (VT). For primary prophylaxis ICD placement was indicated as therapy in patients with low left ventricular ejection fraction (LVEF) $\leq 35 \%$ related do prior myocardial infraction or structural heart disease.

The mean age of the patients was $51.6 \pm 16.4$ years, 9 were men. Four patients had atrial fibrillation (AF) as the basal rhythm, 1 patient - atrial flutter (AFI) and 6 patients - sinus rhythm. One patient had previously implanted cardiac resynchronization therapy (CRT), that was removed during the hospitalization due to infective endocarditis. All patients had at least one condition that precluded the use of a traditional ICD system or had the condition that made the S-ICD more preferable, i.e. a history of previous complicated TV-ICD (18\%), anticipated higher risk of infection (27\%), lack or difficult vascular access (18\%), young age and anticipated high cumulated risk of lifetime device therapy (36\%). Patient characteristics and basic procedural data are summarized in Table 1.

The mean duration of the implantation procedure was $2 \mathrm{~h}$. One patient developed a postoperative pocket hematoma treated conservatively. The same patient experienced severe gastrointestinal bleeding not directly associated with the procedure but related to oral anticoagulant treatment. The complication was treated with endoscopy accompanied with blood transfusion (8 units of packed red blood cells). Mean total time of hospitalization was 28 (6-92) days. The time from admission to the procedure ranged from 1 to 81 days (mean 18 days) and from procedure to discharge from 4 to 27 days (mean 10 days).

During follow-up (mean time $17.5 \pm 9.6$ months) all patients survived. There was no neither infection nor a need for battery replacement but 1 patient needed re-intervention due to in pocket device dislocation (case 3 with subcutaneous device placement). In 3 patients at least one appropriate therapy occurred and 1 patient had an episode of inappropriate therapies due to myopotentials.

The real costs of hospitalization for every patient are presented in Table 1. Average cost of hospitalization per patient was 21,014.29 EUR (minimal $=19,332.71$ EUR and maximal $=24,824.14$ EUR). Average costs for each category were as follows: 18,548.84 EUR for S-ICD, 142.66 EUR for medical materials excluding S-ICD; 142.87 EUR for pharmaceuticals; 157.14 EUR for operating theatre staff; 871.93 EUR for cardiology department staff; 399.33 EUR for laboratory additional tests, 221.13
EUR for non-laboratory additional tests; 521.65 EUR for additional non-medical costs.

\section{Discussion}

The S-ICD has recently entered into the clinical practice and represents a valid alternative for patients who have indications for ICD implantation, especially for those, whose clinical condition makes the risk of traditional TV-ICD implantation notably high. It becomes a less invasive option for patients in whom TV-ICD implantation is surgically impossible. It is estimated that up to $55 \%$ of patients in routine clinical practice needing an ICD are potential candidates for a subcutaneous device $[14,15]$. However, to maximize clinical outcome and cost/benefit ratio, it is fundamental to choose candidates that can benefit most, taking into account both patient and device characteristics.

Based on recently released (2015) Clinical Practice Guidelines for the Prevention of Sudden Cardiac Death of the European Society of Cardiology (ESC), the use of the S-ICD system may be useful, alternatively to the TV-ICD system, when venous access is difficult (especially due to congenital anomalies - writer's note), in young patients facing a lifetime of device therapy and in patients after the removal of a TV-ICD system for infection (Class IIb-C) [2]. Moreover, the device is recommended as an alternative to TV-ICDs when pacing therapy for bradycardia, cardiac resynchronization or antitachycardia pacing is not needed (Class IIa-C) [2]. In recent $2017 \mathrm{AHA} /$ /ACC/HRS Guideline for Management of Patients with Ventricular Arrhythmias and the Prevention of SCD the use of S-ICD has become a Class I-B indication for the mentioned patient group [16]. Because the risk of infection appears to be lower, S-ICD may be preferred in patients who are at high risk of infection, such as those with a prior device infection, end-stage renal failure, diabetes mellitus, or those who are chronically immunosuppressed. This means that majority of ICD indicated patients (without pacing needs) should be considered for SICD according to Class IIa indication endorsed by recent guidelines [17]. Although according to above mentioned recommendations S-ICD can be used in most ICD candidates, due to cost issues in Poland it is possible to introduce this novel therapy only in very selected group of patients. This was reflected in the characteristics of the present study population and may be comparable to recently published data from a European survey showing the following main reasons favoring the use of an S-ICD: young 


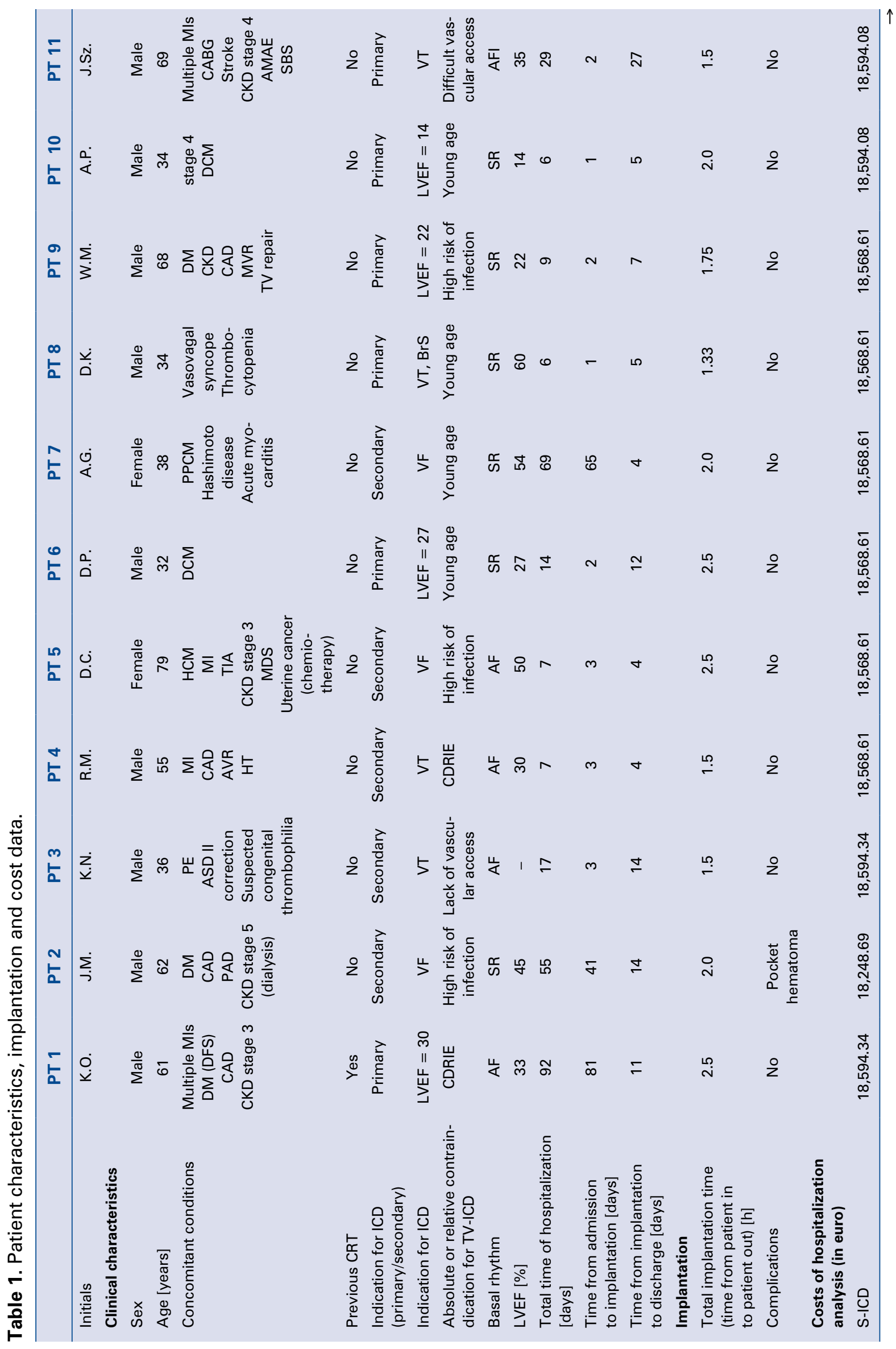




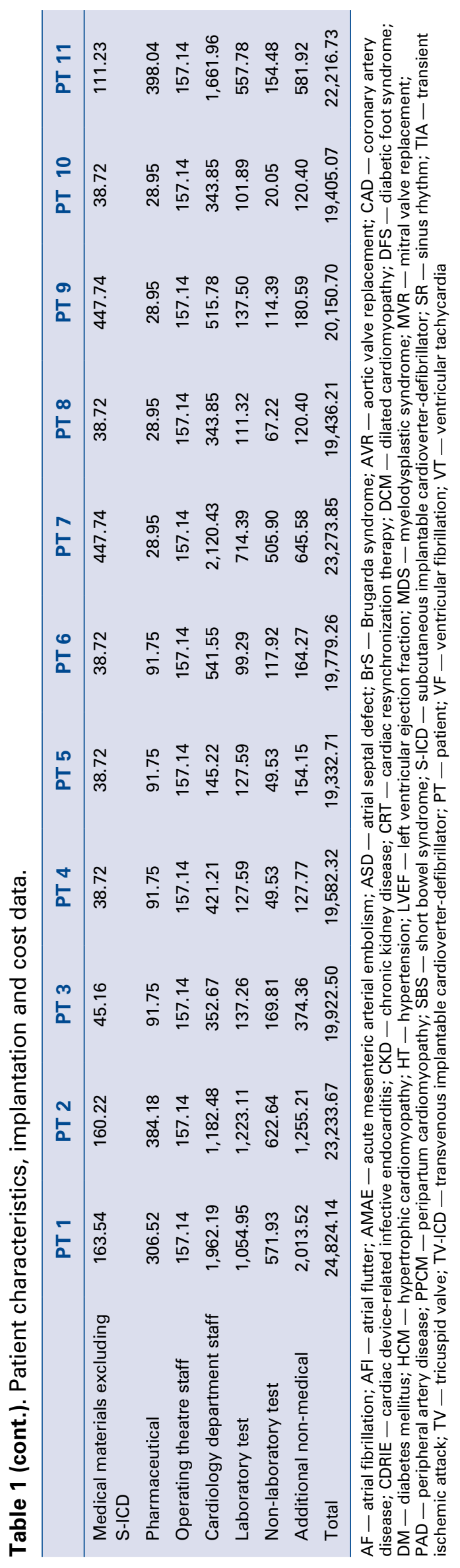

age, lead-related complications, elevated risk or previous device infection, and also patient preference or active lifestyle [18]. In our population 2 patients with the history of cardiac device-related infective endocarditis (CDRIE), the S-ICD was implanted due to increased risk of infection related to the necessity of device re-implantation as well as increased levels of inflammatory parameters during the entire period of hospitalization. Additional risk factors for those patients were: advanced diabetes mellitus complicated by diabetic food syndrome in 1 patient and composite of $\mathrm{CAD}$ and heart valvular disease in the second one.

In 2 patients, TV-ICD implantation was prohibited by venous system anatomy, that made placement of a lead difficult or even impossible or the lack of vascular access associated with the need of intravenous nutrition. Recent studies show that venous abnormalities are not rare among patients requiring pacemaker or ICD therapy and presence of stenosis or obstruction of central veins (in absence of congenital anomalies) can be found in up to $7 \%$ of cases during pre-implant intravenous contrast venography [19], thus further development of this technology can make it first line option in these situations.

Finally, in 4 patients, S-ICD was chosen instead of TV-ICD due to long life expectancy. In those cases, implanting a TV-ICD with an endocardial lead system could put the patient at increased risk of serious systemic and potentially mortal complications such as CDRIE. Here it is worth mentioning that new reports confirmed safety and effectiveness of this system in children [20,21] though implantation in patients with low body mass index should be carefully considered due to the relatively large and heavy generator. Channelopathies, hypertrophic cardiomyopathy (HCM), dilated cardiomyopathy (DCM), are very common problems among young people [22], which is in accord with this study, and those in whom the mechanism of SCD usually involves polymorphic VT or VF, and those with low risk of bradycardia and monomorphic VT requiring Anti Tachycardia Pacing (ATP) theoretically constitutes a group where S-ICD may be the preferable option. However, it should be noted that a higher rate of inappropriate shocks due to T-wave oversensing and double-counting has been recorded in these patients [23, 24]. For example, in patients with Brugada syndrome, although clinical efficacy of S-ICD has been demonstrated, the more recent experience in Europe suggests that S-ICD screening failure occurs in up to $13 \%$ of those patients [24]. Rate of inappropriate S-ICD 
shocks ranges between $4 \%$ and $15 \%,[23,25-27]$ that is not so different from TV-ICD [7, 24], but with a different mechanism: up to $80 \%$ is caused by T-wave oversensing (TWOS), especially in some patient populations (i.e. congenital heart disease, Brugada and long QT syndromes), while in TV-ICD TWOS is involved in about $20 \%$ of cases $[4,5,25$, 26]. This aspect is important because it underlies the importance of exercise testing shortly after the implantation, as well as adopting a detailed patient screening practice with selection of the optimal sensing vector in different positions to reduce the risk of TWOS [28, 29]. Careful screening, software updates and template selection has reduced the occurrence of inappropriate shocks with the current S-ICDs when compared with the earlier experiences [29].

Another concern that has been raised is that there was no pacing capability (with the exception of 30" post-shock backup pacing) within S-ICD therapy which also means no possibility to deliver ATP for management of refractory monomorphic VT. This aspect was crucial in deciding on S-ICD implantation in the last patient in whom the benefits of S-ICD implantation (central venous circulation protection, avoidance of the lead related complications etc.) outweighed the lack of ATP function. The SCD-HeFT study showed that after almost 3 years of follow-up, only a third of patients with VT had more than one episode, which translated to $1.8 \%$ annual risk [30,31]. In one cohort of patients it was shown that $55.5 \%$ (95\% confidence interval $52.0-59.0 \%$ ) of single or dual chamber ICDs recipients did not require pacing or receive ATP after 5 years and therefore could have been eligible to receive an S-ICD instead. The Pacing Fast Ventricular Tachycardia Reduces Shock Therapies (Pain-FREE Rx II) trial [32] showed that patients randomized to receive shock-only, $34 \%$ of fast VT episodes terminated spontaneously before therapy, suggesting that a considerable proportion of ATP intervention, which is delivered before a shock, may be unnecessary. Furthermore, ATP is not without risk; it is possible for ATP to accelerate VT to polymorphic VT or VF [33-35] which has been associated with a higher mortality risk [36]. In conclusion, the lack of ATP function in the S-ICD is a contentious one [37].

According to the latest reports of the current use of S-ICDs across a broad range of European centers, the most reported reasons for preferring an S-ICD over implantation of a TV-ICD are: an anticipated difficult vascular access (82\% of the centers), a history of previous complicated TV-ICD (80\% of the centers), young age (69\%), an anticipated higher risk of infection (63\%), the availability of the new generation S-ICD (53\% of the centers), or a primary prevention indication ( $45 \%$ of the centers) [10]. In the present study the most common indication for S-ICD implantation was the young age of patients facing a lifetime of device therapy thus avoiding the lead-related complications (40\% of patients), what can be explained by new reports demonstrated that lead failure rates are higher in younger patients [25, 38].

In the aforementioned study, more than onefourth $(\mathrm{n}=14,27 \%)$ of the respondents have never implanted an S-ICD. The main reasons reported by these centers for the non-use of S-ICD include economic issues, such as lack of reimbursement (25\% were not implanting centers), high cost of the device (25\% of centers) and less often claimed issues were the lack of training (19\% of centers), the complexity of the implantation procedure (6\% of centers) [10]. Those results also reflect limitations in S-ICD implantation, that started in the documented hospital from 2015 [39]. Although implantation of TV-ICD is on the list of guaranteed services, total cost of S-ICD highly exceeds reimbursement level for this category, so individual financing was implemented for each patient and this paper depicts expenditures divided into range of categories. In the Honarbakhsh et al. [40] study, comparing efficacy, safety and costs of the S-ICD vs. TV-ICD, even though more expensive, S-ICD was associated with a relative risk reduction of device-related complication of $70 \%$ with a hazard risk of 0.30 (95\% confidence interval $0.12-0.76$; $\mathrm{p}=0.01$ ) compared to TV-ICDs. The total mean cost for each group, including the complication-related costs was $£ 9967 \pm 4511(\$ 13,639 \pm 6173)$ and $£ 12,601 \pm 1786(\$ 17,243 \pm 2444)$ in the TV-ICD and S-ICD groups respectively $(\mathrm{p}=0.0001)$ [19]. It shows that despite the existing significant difference in unit cost of the S-ICD, overall S-ICD costs may be mitigated versus TV-ICDs over a longer followup period. If the incidence rate of TV-ICD-related complications [40] remains stable over the next years no cost difference between these two groups would be expected.

Until now, there is still relatively little data regarding long term performance of the S-ICD in a "real world" setting so larger experience and longer follow up are required, but initial results are encouraging [41, 42]. 


\section{Limitations of the study}

This single center experience was not randomized and shows the short term, initial experience of the present small group of patients. This implies that no real data on long-term performance of the device and its longevity were available. A low number of patients in the present cohort is the main limitation of the above analysis. However, the aim of the analysis was to present the initial experience of the operating team with the new treatment method in a single center and to present full hospitalization costs of "real-world" consecutive patients. Such data might be valuable for regional authorities to support decisions on reimbursement and for appropriate valuations of the procedure.

\section{Conclusions}

Current evidence suggests that S-ICD is a highly effective and safe modality with comparable defibrillation success rate to conventional ICDs. Thus, a wide range of patients without pacing requirements, and particularly younger patients, may benefit. Ongoing clinical studies will help establish the S-ICD system long-term safety and efficacy and better define target patient groups.

Conflict of interest: Marcin Grabowski, Andrzej Cacko and Marcin Michalak report receiving lecture honoraria from Boston Scientific.

\section{References}

1. Pagidipati NJ, Gaziano TA. Estimating deaths from cardiovascular disease: a review of global methodologies of mortality measurement. Circulation. 2013; 127(6): 749-756, doi: 10.1161/CIRCULATIONAHA.112.128413, indexed in Pubmed: 23401116.

2. Priori SG, Blomström-Lundqvist C, Mazzanti A, et al. 2015 ESC Guidelines for the management of patients with ventricular arrhythmias and the prevention of sudden cardiac death: The Task Force for the Management of Patients with Ventricular Arrhythmias and the Prevention of Sudden Cardiac Death of the European Society of Cardiology (ESC)Endorsed by: Association for European Paediatric and Congenital Cardiology (AEPC). Eur Heart J. 2015; 36(41): 2793-2867, doi: 10.1093/eurheartj/ehv316, indexed in Pubmed: 26320108.

3. Jacheć W, Tomasik A, Polewczyk A, et al. Impact of ICD lead on the system durability, predictors of long-term survival following ICD system extraction. Pacing Clin Electrophysiol. 2017; 40(10): 1139-1146, doi: 10.1111/pace.13173, indexed in Pubmed: 28846144.

4. Aydin A, Hartel F, Schlüter M, et al. Shock efficacy of subcutaneous implantable cardioverter-defibrillator for prevention of sudden cardiac death: initial multicenter experience. Circ Arrhythm Electrophysiol. 2012; 5(5): 913-919, doi: 10.1161/CIRCEP.112.973339, indexed in Pubmed: 22923274.
5. Köbe J, Reinke F, Meyer C, et al. Implantation and follow-up of totally subcutaneous versus conventional implantable cardioverter-defibrillators: a multicenter case-control study. Heart Rhythm. 2013; 10(1): 29-36, doi: 10.1016/j.hrthm.2012.09.126, indexed in Pubmed: 23032867.

6. Dabiri Abkenari L, Theuns DA, Valk SDA, et al. Clinical experience with a novel subcutaneous implantable defibrillator system in a single center. Clin Res Cardiol. 2011; 100(9): 737-744, doi: 10.1007/s00392-011-0303-6, indexed in Pubmed: 21416191.

7. Olde Nordkamp LRA, Dabiri Abkenari L, Boersma LVA, et al. The entirely subcutaneous implantable cardioverter-defibrillator: initial clinical experience in a large Dutch cohort. J Am Coll Cardiol. 2012; 60(19): 1933-1939, doi: 10.1016/j.jacc.2012.06.053, indexed in Pubmed: 23062537.

8. Jarman JWE, Lascelles K, Wong T, et al. Clinical experience of entirely subcutaneous implantable cardioverter-defibrillators in children and adults: cause for caution. Eur Heart J. 2012; 33(11): 1351-1359, doi: 10.1093/eurheartj/ehs017, indexed in Pubmed: 22408031.

9. Weiss R, Knight BP, Gold MR, et al. Safety and efficacy of a totally subcutaneous implantable-cardioverter defibrillator. Circulation. 2013; 128(9): 944-953, doi: 10.1161/CIRCULATIONAHA.113.003042, indexed in Pubmed: 23979626.

10. Boveda S, Lenarczyk R, Haugaa K, et al. Implantation of subcutaneous implantable cardioverter defibrillators in Europe: results of the European Heart Rhythm Association survey. Europace. 2016; 18(9): 1434-1439, doi: 10.1093/europace/euw258, indexed in Pubmed: 27582309.

11. Ptaszyński P, Grabowski M, Kowalski O, et al. Opinia Sekcji Rytmu Serca Polskiego Towarzystwa Kardiologicznego dotycząca zastosowania podskórnego kardiowertera-defibrylatora w prewencji nagłego zgonu sercowego w warunkach polskich. Kardiol Pol. 2017; 75(10): 1057-1060, doi: 10.5603/kp.2017.0196, indexed in Pubmed: 29057442.

12. Bordachar P, Marquié C, Pospiech T, et al. Subcutaneous implantable cardioverter defibrillators in children, young adults and patients with congenital heart disease. Int J Cardiol. 2016; 203: 251-258, doi: 10.1016/j.ijcard.2015.09.083, indexed in Pubmed: 26519678 .

13. Akerström F, Arias MA, Pachón M, et al. The Entirely Subcutaneous Defibrillator (S-Icd): State of the Art and Selection of the Ideal Candidate. World J Cardiol. 2013; 5(9): 347-354.

14. Grace A. The subcutaneous implantable cardioverter-defibrillator. Curr Opin Cardiol. 2010; 29(1): 10-19, doi: 10.1097/ HCO.0000000000000031, indexed in Pubmed: 24284982.

15. de Bie MK, Thijssen J, van Rees JB, et al. Suitability for subcutaneous defibrillator implantation: results based on data from routine clinical practice. Heart. 2013; 99(14): 1018-1023, doi: 10.1136/heartjnl-2012-303349, indexed in Pubmed: 23704324.

16. Al-Khatib S, Stevenson W, Ackerman M, et al. 2017 AHA/ACC/ /HRS Guideline for Management of Patients With Ventricular Arrhythmias and the Prevention of Sudden Cardiac Death: Executive Summary. Heart Rhythm. 2017, doi: 10.1016/j. hrthm.2017.10.035.

17. Friedman DJ, Parzynski CS, Varosy PD, et al. Trends and InHospital Outcomes Associated With Adoption of the Subcutaneous Implantable Cardioverter Defibrillator in the United States. JAMA Cardiol. 2016; 1(8): 900-911, doi: 10.1001/jamacardio.2016.2782, indexed in Pubmed: 27603935. 
18. Boveda S, Lenarczyk R, Fumagalli S, et al. Factors influencing the use of subcutaneous or transvenous implantable cardioverter-defibrillators: results of the European Heart Rhythm Association prospective survey. Europace. 2018 [Epub ahead of print], doi: 10.1093/europace/euy009, indexed in Pubmed: 29432525.

19. Korkeila P, Nyman K, Ylitalo A, et al. Venous obstruction after pacemaker implantation. Pacing Clin Electrophysiol. 2007; 30(2): 199-206, doi: 10.1111/j.1540-8159.2007.00650.x, indexed in Pubmed: 17338716.

20. Poole JE, Gold MR. Who should receive the subcutaneous implanted defibrillator?: The subcutaneous implantable cardioverter defibrillator (ICD) should be considered in all ICD patients who do not require pacing. Circ Arrhythm Electrophysiol. 2013; 6(6): 1236-44; discussion 1244, doi: 10.1161/CIRCEP.113.000481, indexed in Pubmed: 24347599.

21. Berul CI, Van Hare GF, Kertesz NJ, et al. Results of a multicenter retrospective implantable cardioverter-defibrillator registry of pediatric and congenital heart disease patients. J Am Coll Cardiol. 2008; 51(17): 1685-1691, doi: 10.1016/j.jacc.2008.01.033, indexed in Pubmed: 18436121.

22. Janson CM, Patel AR, Bonney WJ, et al. Implantable cardioverter-defibrillator lead failure in children and young adults: a matter of lead diameter or lead design? J Am Coll Cardiol. 2014; 63(2): 133-140, doi: 10.1016/j.jacc.2013.09.033, indexed in Pubmed: 24140671.

23. Jarman JWE, Todd DM. United Kingdom national experience of entirely subcutaneous implantable cardioverter-defibrillator technology: important lessons to learn. Europace. 2013; 15(8): 1158-1165, doi: 10.1093/europace/eut016, indexed in Pubmed: 23449924.

24. Conte G, Kawabata M, de Asmundis C, et al. High rate of subcutaneous implantable cardioverter-defibrillator sensing screening failure in patients with Brugada syndrome: a comparison with other inherited primary arrhythmia syndromes. Europace. 2017 [Epub ahead of print], doi: 10.1093/europace/eux009, indexed in Pubmed: 28340026.

25. Ascoeta MS, Marijon E, Defaye P, et al. Impact of early complications on outcomes in patients with implantable cardioverterdefibrillator for primary prevention. Heart Rhythm. 2016; 13(5): 1045-1051, doi: 10.1016/j.hrthm.2015.12.046, indexed in Pubmed: 26749313.

26. Olde Nordkamp LRA, Postema PG, Knops RE, et al. Implantable cardioverter-defibrillator harm in young patients with inherited arrhythmia syndromes: A systematic review and meta-analysis of inappropriate shocks and complications. Heart Rhythm. 2016; 13(2): 443-454, doi: 10.1016/j.hrthm.2015.09.010, indexed in Pubmed: 26385533.

27. Kooiman KM, Knops RE, Olde Nordkamp L, et al. Inappropriate subcutaneous implantable cardioverter-defibrillator shocks due to T-wave oversensing can be prevented: implications for management. Heart Rhythm. 2014; 11(3): 426-434, doi: 10.1016/j. hrthm.2013.12.007, indexed in Pubmed: 24321235.

28. Lewis GF, Gold MR. Safety and Efficacy of the Subcutaneous Implantable Defibrillator. J Am Coll Cardiol. 2016; 67(4): 445-454, doi: 10.1016/j.jacc.2015.11.026, indexed in Pubmed: 26821634.

29. Brisben AJ, Burke MC, Knight BP, et al. A new algorithm to reduce inappropriate therapy in the S-ICD system. J Cardiovasc Electrophysiol. 2015; 26(4): 417-423, doi: 10.1111/jce.12612, indexed in Pubmed: 25581303.

30. Poole JE, Johnson GW, Hellkamp AS, et al. Prognostic importance of defibrillator shocks in patients with heart failure. $\mathrm{N} \mathrm{Engl}$ J Med. 2008; 359(10): 1009-1017, doi: 10.1056/NEJMoa071098, indexed in Pubmed: 18768944.
31. Hanna R, Hellkamp A, Mark D, et al. Predictors of ventricular tachyarrhythmias treated with the ICD in the sudden cardiac death in heart failure trial. Poster, ESC Congress 2012. Munich, Germany. August; 26: 2012.

32. Wathen MS, DeGroot PJ, Sweeney MO, et al. Prospective randomized multicenter trial of empirical antitachycardia pacing versus shocks for spontaneous rapid ventricular tachycardia in patients with implantable cardioverter-defibrillators: Pacing Fast Ventricular Tachycardia Reduces Shock Therapies (PainFREE Rx II) trial results. Circulation. 2004; 110(17): 2591-2596, doi: 10.1161/01.CIR.0000145610.64014.E4, indexed in Pubmed: 15492306 .

33. Saxon LA. The subcutaneous implantable defibrillator: a new technology that raises an existential question for the implantable cardioverter-defibrillator. Circulation. 2013; 128(9): 938-940, doi: 10.1161/CIRCULATIONAHA.113.004794, indexed in Pubmed: 23979625.

34. Sweeney MO, Wathen MS, Volosin K, et al. Appropriate and inappropriate ventricular therapies, quality of life, and mortality among primary and secondary prevention implantable cardioverter defibrillator patients: results from the Pacing Fast VT REduces Shock ThErapies (PainFREE Rx II) trial. Circulation. 2005; 111(22): 2898-2905, doi: 10.1161/CIRCULATIONAHA.104.526673, indexed in Pubmed: 15927965.

35. Wathen MS, Sweeney MO, DeGroot PJ, et al. PainFREE Investigators. Shock reduction using antitachycardia pacing for spontaneous rapid ventricular tachycardia in patients with coronary artery disease. Circulation. 2001; 104(7): 796-801, indexed in Pubmed: 11502705.

36. Young JB, Abraham WT, Smith AL, et al. Combined cardiac resynchronization and implantable cardioversion defibrillation in advanced chronic heart failure: the MIRACLE ICD Trial. JAMA. 2003; 289(20): 2685-2694, doi: 10.1001/jama.289.20.2685, indexed in Pubmed: 12771115.

37. Patel KH, Lambiase PD. The subcutaneous ICD - current evidence and challenges. Cardiovasc Diagn Ther. 2014; 4(6): 449-459, doi: 10.3978/j.issn.2223-3652.2014.12.02, indexed in Pubmed: 25610802.

38. Chue CD, Kwok CS, Wong CW, et al. Efficacy and safety of the subcutaneous implantable cardioverter defibrillator: a systematic review. Heart. 2017; 103(17): 1315-1322, doi: 10.1136/ heartjnl-2016-310852, indexed in Pubmed: 28687562.

39. Kowara M, Michalak M, Cacko A, et al. First experience with a subcutaneous implantable cardioverter-defibrillator (S-ICD) implantation - a case series study of single Cardiology Center. Heart Beat J. 2016; 1: 18-23, doi: 10.24255/hbj/67039.

40. Honarbakhsh S, Providencia R, Srinivasan N, et al. A propensity matched case-control study comparing efficacy, safety and costs of the subcutaneous vs. transvenous implantable cardioverter defibrillator. Int J Cardiol. 2017; 228: 280-285, doi: 10.1016/j. ijcard.2016.11.017, indexed in Pubmed: 27865198.

41. Lambiase PD, Barr C, Theuns DA, et al. EFFORTLESS Investigators. Worldwide experience with a totally subcutaneous implantable defibrillator: early results from the EFFORTLESS S-ICD Registry. Eur Heart J. 2014; 35(25): 1657-1665, doi: 10.1093/eurheartj/ehu112, indexed in Pubmed: 24670710.

42. Pedersen SS, Lambiase P, Boersma LVA, et al. Evaluation oF FactORs ImpacTing CLinical Outcome and Cost EffectiveneSS of the S-ICD: design and rationale of the EFFORTLESS S-ICD Registry. Pacing Clin Electrophysiol. 2012; 35(5): 574-579, doi: 10.1111/j.1540-8159.2012.03337.x, indexed in Pubmed: 22360677. 